\title{
Economic Development in Mexico and the Role of Blockchain
}

\author{
Fabian Zbinden, Galia Kondova* \\ School of Business, University of Applied Sciences and Arts (FHNW), Switzerland
}

Copyright $\bigcirc 2019$ by authors, all rights reserved. Authors agree that this article remains permanently open access under the terms of the Creative Commons Attribution License 4.0 International License

\begin{abstract}
This paper studies recent economic and political developments in Mexico and the potential role of blockchain technology in improving governance and fighting corruption in the country. Mexico badly needs innovative solutions to fight the persistent problems of corruption and inefficient governance. At the same time, blockchain, being a decentralized technology, promises to reduce administrative costs and ensure transparency in the public services. The paper takes stock of the current state of blockchain implementation in the economy and in the public administration of Mexico as well as studies the advantages and disadvantages related to the further outspread of the technology in the country. Moreover, the work aims at drawing policy recommendations as to the future role of blockchain in the public management and economic development in Mexico.
\end{abstract}

Keywords Mexico, Development Economics, Blockchain, Public Services

\section{Introduction}

In September 2017, the Mexican government has initiated a blockchain project called "Blockchain HACKMX". The project's main goal is to "promote government digital innovation" and to address the issue of public contract corruption [1].

Moreover, at the World Economic Forum 2018 international experts openly discussed the question "Can blockchain help in the fight against corruption?" [2]. Some of the expressed opinions stress out that blockchain "could add a layer of security to records and transactions that are particularly exposed to high corruption risks" but "it will not replace the need for stronger institutions" [2].

The Mexican senate approved in March 2018 a new law concerning financial technologies, including a framework for digital assets, which should provide security and clarity to retail investors, debtors as well as institutions. This bill (Fintech Law) is considered to lay an important foundation for the whole crypto and blockchain market in Mexico [3].

Considering these developments, the paper provides a stocktaking of the current outspread of blockchain in Mexico accompanied by an in-depth analysis of the advantages and disadvantages associated with the technology. Moreover, policy recommendations are drawn as to the further implementation of blockchain in the country. Thus, the study is the first one to the best knowledge of the authors to provide such a comprehensive analysis on blockchain in Mexico.

The paper starts with a literature overview on the blockchain technology as well as on the recent economic and political developments in Mexico. Then a comprehensive overview of the current outspread of blockchain in Mexico is provided. Finally, the potential advantages and disadvantages of blockchain for the country are discussed on the basis of which important policy recommendations are formulated.

\section{Literature Review}

\section{Blockchain and Smart Contracts}

Blockchain is a programmed digital ledger technology (DLT) based on cryptography. This digital ledger can to a certain extent be compared to a traditional database. Unlike a common centralized database, the network of a distributed ledger does not rely on one or a few central instances [4]. Instead, blockchain functions as a decentralized and distributed network (see Exhibit 1). This implies that the entire network must be operated by a multitude of servers, referred to as nodes. Since this network is decentralized, a special consensus mechanism is required to ensure the authenticity, as well as the integrity of the data [4]. 


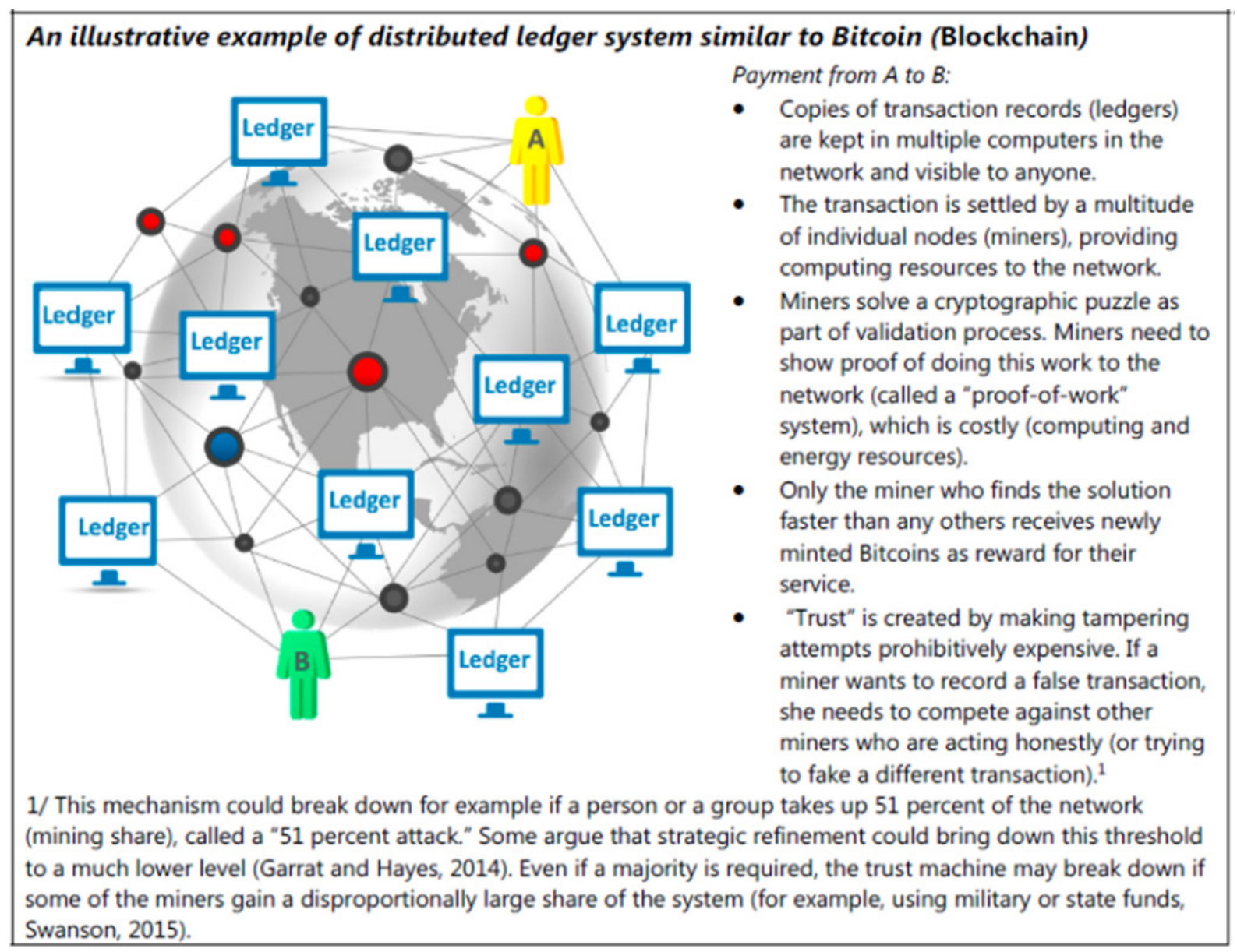

Source: He et al. (IMF Staff Discussion Notes SDN/16/03)

Exhibit 1. The Blockchain Technology

Kostarev [5] argues that consensus among all operating nodes is a crucial factor for the functionality and the security of a blockchain. To ensure that this mechanism works properly, it is of particular importance that it has to be powered by a collective self-interest shared among all nodes. This can be achieved by creating a network, which is more profitable to protect than to hack. Ultimately, it becomes clear that with a well-established consensus mechanism it is very unlikely that one person would be able to corrupt, manipulate or even take down the entire network [5].

Furthermore, Bradley [6] describes blockchain as an autonomous functional network, which successfully manages to cut out the middleman as compared to a centralized system. Thus, people are not required to trust any intermediary in order to exchange data globally. At the same time, the data integrity as well as its security remains ensured. This results in many advantages, such as high transparency and rapid settlement [6]. Tapscott \& Tapscott [7] even praise the blockchain technology as the first fully trustworthy peer-to-peer transaction network. This may be explained by the fact that blockchain is not powered by large corporations and their pure interest in profit.

The practical application of the blockchain technology was introduced by a self-published paper of an author called Satoshi Nakamoto in October 2008. Nakamoto [8] published a protocol for a purely peer-to-peer electronic cash system called Bitcoin. According to Nakamoto [8] the idea of Bitcoin was to create "an electronic payment system based on cryptographic proof instead of trust, allowing any two willing parties to transact directly with each other without the need for a trusted third party". The crypto technology used behind Bitcoin is a special type of DLT and commonly referred to as blockchain [8].

Nakamoto [8] established a set of parameters including a fixed maximum supply of 21 million Bitcoins as well as a rate of inflation. These parameters were implied into the protocol at the very beginning and they are being continuously enforced by network participants (nodes) today. Today the Bitcoin network is run and supported by a vast number of nodes which are distributed all around the globe. This distributed network is typically referred to as blockchain.

The majority of these nodes are miners as well, which means they create new bitcoins. At the same time, the miners fulfil a crucial task of ensuring consensus for the network. Nakamoto [8] argues that already existing digital signatures are only the first part of a pure peer-to-peer 
network. To ensure a fully functional system without the necessity of a third central party one must solve the key issue called the double-spending problem [9]. Since Bitcoins only exist as a digital coin, it could be duplicated or counterfeit.

In order to protect the network from fraud, Nakamoto [8] added a so-called Proof-of-Work (PoW) consensus mechanism to the formula. As the name blockchain already implies, data get stored in blocks, which then are built on top of each other, forming a continuously expanding chain. In order to find a block, miners use advanced computer power to solve a complex mathematical puzzle. Once a miner solved the puzzle by finding the correct answer, he proofs that he has done the required necessary work. This proof delegates him now to take recent unconfirmed transactions and arrange them into a block. Simultaneously he validates all legitimate transactions making sure that no double spending occurs. Eventually this block is propagated to the entire network so other miners can confirm the transactions as legitimate. As soon as the block is completed, miners get rewarded with new bitcoins, therefore creating new ones. This process recurs approximately every 10 minutes.

Briefly, the blockchain technology allows information (e.g. transactions) to be permanently recorded in blocks, which build an immutable chain once they are confirmed. Afterwards this information cannot be overwritten, deleted or altered in any way. Conclusively, a combination of cryptography, consensus mechanism and incentives for miners replace the need of a central authority acting as a controlling body. Eventually it allows Bitcoin to function as a decentralized and secure network.

Furthermore, there are different types of blockchains with regard to the network participants and the access to the blockchain. On the one hand, there are the fully public blockchains such as the Bitcoin, Ethereum or Waves network. These systems are built on the principle of a decentralized network, which is accessible to every internet user. To ensure the flawless functionality of the system, cryptographic mechanisms combined with economic incentives are coded into the protocol [4]. To secure the network, public blockchains embed own coins, to reward the nodes running the network, and use different consensus mechanisms such as PoW or Proof of Stake (PoS). PoS is a less energy intensive alternative to PoW that does not require advanced computation power. Instead, the PoS protocol is based on the principles of a lottery where the stake of coins held by each node determines the likelihood of being chosen to execute the computation and thus add the next block to the chain [5].

On the other hand, there are private and permissioned blockchains. Fully private blockchains do not require any coins or complicated consensus mechanisms to secure the network, since all nodes are posed by the same entity. Since the network is used for a private environment, it acts like a centralized database, meaning that one entity controls the network and determines which data is added to the blockchain. Therefore, there is still a single point of failure and control within such systems.

In addition, there are hybrid or the so-called permissioned blockchains. These are run by a restricted number of pre-selected entities. Accessibility is also restricted to chosen persons, organizations or a certain consortium. As the word hybrid implies, such systems have public as well as private aspects. Also, the degree of decentralization depends on the architecture of the network, including the number of nodes and the power of control which one entity holds within the network [4].

Smart Contracts are the necessary tools to obviate the need of any trusted intermediaries and therefore making blockchain-based economy possible. A smart contract is written as a digital script, which allows its creator(s) to incorporate reliable contract clauses. They differ from normal contracts because they are self-executing and solely exist in a digital form on the blockchain. Although most importantly their functioning is based on reliable and autonomous mechanisms which provides fast and secure settlements. This means that digital value will get exchanged only if certain conditions are fulfilled - all verified by the blockchain. Thanks to the decentralized governance of a blockchain, smart contracts are transparent and ensure an autonomous execution. Once a smart contract is deployed on the blockchain, it becomes immutable. Of course, the contractor(s) may include certain rules, which enables modifications [10].

With smart contracts, the scope of blockchain applications reach far beyond cryptocurrencies [11]. Experts expect that the technology will eventually disrupt many industries, such as healthcare, real estate, governance or energy distribution [12]. Likewise, the Swiss Blockchain Taskforce [13] holds the view that "the political dimension of blockchain technology is immense, even epoch-defining". The taskforce is convinced that Blockchain can lay the crucial technical foundation for a decentralized peer- to- peer economy.

\section{Advantages of Decentralization and the Blockchain Technology}

Transparency is considered to be one of the great advantages associated with the application of blockchain [14]. Networked integrity is believed to be another [7]. Given that the unique architecture of a blockchain prevents any act of dishonesty, it is impossible to fool users as well as to falsify data [7].

Tapscott \& Tapscott [7] argue that blockchain protocols are not only designed to enable full user and data integrity, but also to create path-breaking governance methods. Blockchain protocols reduce the centralization of power by handing it over to all network participants. If a single authority tries to abuse its power, the whole network can see what is happening thus keeping control by isolating the 
malicious party from the network. Transparent records of all information and metadata facilitates this power distribution in the first place. Finally, blockchain introduced a revolutionary way of distributing political power, which is more transparent, and user friendly than all existing governance systems [7].

Blockchain provides enhanced security and privacy through its decentralized nature. As illustrated in Tapscott \& Tapscott [7], everybody participating in the network is required to use cryptographic tools. As a result, blockchain offers higher data protection than common firewalls could ever provide and keeps users' personal information private.

\section{Disadvantages and Technical Challenges}

Although blockchain offers promising solutions for many issues, there are also disadvantages and challenges associated with the technology.

There are concerns associated with the lack of standardization in decentralized systems [14]. Due to the complex consensus mechanism in public as well as private blockchains, maintenance of the system might be costlier and riskier as well as inflexible to change in comparison to traditional database systems. The implementation of even minor changes requires the majority of the nodes to decide, i.e. vote, for the activation of the new features. This consensus-driven process takes longer in the case of a blockchain architecture than it would take with traditional databases. Moreover, in the presence of technical disunity among the nodes, the blockchain might be split into two separate chains [15].

The degree of decentralization is a major factor in understanding the complexity of the blockchain technology. Although many blockchain projects strive to become "fully" decentralized, they find it very hard to reach a high level of decentralization [16]. The higher the level of decentralization, i.e. the higher the number of independent nodes running the system, the more secure the system is. Unfortunately, a higher degree of decentralization always entails more drawbacks such as complex development and scaling issues. Consequently, there are arguments that the future of blockchain applications will rather be a mixture of centralized and decentralized elements [14].

The high energy consumption is another issue associated with the blockchain technology [17]. Since many cryptocurrencies, including Bitcoin, use a PoW mechanism to achieve consensus among the network, their blockchains rely on a high amount of computing power and thus a high energy consumption. Digiconomist [18] reports Bitcoin's estimated energy consumption to be $73.12 \mathrm{TWh}$ annually. This amount is comparable to the annual electricity consumption of a country like Austria [19].

On the other hand, Antonopoulos [20] argues that the «energy consumption problem» is often misrepresented. The author argues that the Bitcoin energy consumption debate should be put in relation to the energy consumption required for credit cards use, datacenters, tower offices, trading floors, bank vaults, etc. instead. This view is supported by Domingo [21] who estimated that energy consumption from banks amount to 100TWh annually. He also expects Bitcoins efficiency to further increase due to technical developments in the field of hardware (mining rigs) and software (protocols).

Furthermore, Antonopoulos [20] argues that Bitcoin might even end up as the most energy efficient financial system on a global scale. He points out that the use of energy required to mine Bitcoins is geographical independent. Therefore, Bitcoin actually contributes to the decentralization of energy and boosts the trend of improving renewable and alternative energy. Although Antonopoulos [20] takes a much less critical stand on Bitcoins energy consumption, he believes that the world cannot afford more than one PoW systems. Many blockchain businesses are constantly trying to improve their technology, while different consensus mechanisms are adopted. As described by Kostarev [5], there are other forms of consensus mechanism, which are less costly as well as less energy intensive. Proof-of-Stake (PoS) is just one alternative approach, which has been adopted by various blockchain projects. The main difference lies in the hardware, which is required to participate in the network (i.e. run a node). In PoS system, only the stake of coins held by participants determine the likelihood of who will add the next block of transactions to the chain. Consequently, the PoS consensus mechanism does not require huge computing power and therefore no costly hardware to run a node.

Another widely discussed issue related to cryptocurrencies is the risk of money laundering and cybercrime. Authorities worldwide are concerned that the decentralized and semi-anonymous nature of cryptocurrencies might be used for financing criminal activities. In addition, in a report released in May 2018, the Anti-Phishing Working Group reports that cryptocurrencies worth about 1.2 billion USD were stolen since 2017 [22].

\section{Economic, Political and Social Developments in Mexico}

The 2017 OECD Economic Survey for Mexico provides the following assessment of the country:

"Mexico is now the world's 11th largest economy (in terms of GDP measured at purchasing power parity). The country has gone through tremendous structural changes over the past three decades. From an oil-dependent economy up to the early 1990s to a booming manufacturing centre in the aftermath of NAFTA in the mid-1990s, Mexico is now increasingly becoming an international trade hub. The proximity to the US export market continues to be a competitive advantage, but Mexico has strategically boosted free trade, signing 12 agreements with 46 countries. Mexico is now a top global exporter of cars and 
flat screen TVs, among other products. Yet, Mexico's economic potential has been hindered by important challenges such as high levels of poverty, ex-tensive informality, low female participation rates, insufficient educational achievement, financial exclusion, weak rule of law, and persistent levels of corruption and crime" [23].

\section{Poverty}

Estevez [24] reports that only 15 people practically own almost $5 \%$ of Mexico's wealth. At the same time, $43.6 \%$ of Mexican people are living below the national poverty line [25]. This massively imbalanced wealth distribution speaks for itself and is reflected in many statistics.

Compared to the other OECD countries, Mexico scores highest at income inequality and its average wages are at the lowest rate [26, 27]. More troubling is the fact that these inequality trends have been relatively stable in Mexico over the past 20 years [28].

In 2005, the World Bank published a report, which states that less than $25 \%$ of Mexico City's population uses formal financial services. Ten years later, however, it is reported that $44 \%$ of all Mexicans have access to a bank account [29]. These data clearly show that Mexico already has been doing some progress to reduce financial exclusion. Nevertheless, access to the financial services like bank loans or microlending is still considered as weak [30].

Interestingly, this denial of access is not primarily caused due to the lack of financial institutions in rural areas. Instead, high fees, distrust of banks and poor documentation have been among the main reasons reported by participants of a survey [31].

According to De Soto [32], the financial and thus economic exclusion is closely associated with the poor documentation in developing countries. Only for real estate, he estimates the total worth of this caused "dead capital" to amount to at least 9.3 trillion USD. Missing or improper legal documentation, ranging from identity records to economic activities and unrecorded ownerships, represents an enormous obstacle for poor people to escape the "informal economy". For example, one main reason is that people cannot use their property as collateral to apply for a bank credit. These finding is confirmed by the results of a survey, which revealed that although $63 \%$ of unbanked people living in Mexico City own their houses, they do not legally own them due to the lack of legal documentation [31].

\section{Corruption}

In addition to poverty, another great problem in Mexico is the corruption on government level as well as in other public institutions. According to Transparency International [33], Mexico only scores 29 points on the corruption perception index, where 0 represents "highly corrupt" and 100 "very clean". The perceived level of corruption today is at its highest within the last twenty years [33]. Not surprisingly, more than 8 out of 10 Mexicans perceive their political leaders as corrupt [34].

As previously stated, most of the Mexican people do not have faith in their own government. The massive abuse of power on multiple levels also affects social trust in a society. Looking at the results of the Pew Global Attitudes Project, it is of no surprise that Mexico's public satisfaction is at its lowest since 2007 [34]. At the same time, several studies have recognized a strong positive correlation between economic growth and social trust $[35,36]$.

\section{Blockchain Developments in Mexico}

\section{Regulatory Framework}

The new regulatory bill "Ley para Regular las Instituciones de Tecnología Financiera" (Mexico Fintech Law) on Fintech companies, including firms operating in the field of cryptocurrency, was approved by the Mexican congress in March 2018. This bill generally aims at greater regulatory certainty within the fast growing fintech sector in Mexico by providing more guidelines for electronic payments, crowdfunding and digital assets [37]. The new law does not include specific details about the regulation of Bitcoin or other cryptocurrencies itself though. Nevertheless, it provides a conceptual legal framework for digital assets and therefore might be particularly valued by firms interacting with cryptocurrencies. One important aspect is that cryptocurrencies are now recognized as digital assets and thus as a legitimate mean of digital payments and transactions [38]. This is crucial for the entire industry, notably it favors companies operating in the e-commerce and the remittance sector.

Another part of the legislation targets cryptocurrency exchanges directly by putting the Mexican central bank in charge as a regulatory body. This means that exchanges rely on the central bank approval to operate. Furthermore, the law grants the central bank authority to define and determine the scope of permitted virtual activities, such as the listing of cryptocurrencies on exchanges [38]. This control imposed by the central bank might cause a conflict of interest in the crypto community. On the one hand, it aims to prevent money laundering, terrorist organizations and prevent corruption. On the other hand, it might retard the development of Mexicans crypto industry and consequently, exchanges as well as other firms using digital assets would move to countries with a more liberal regulation environment. The authorities are in the process of drafting secondary regulations with specific guidelines on the implementation of the law [39].

With the Fintech Law, Mexico put itself among very few countries, which are proactively establishing laws for the fintech industry [37]. In fact, Mexico became the first country in Latin America to specifically regulate this 
industry [40]. Since the new approved law aims to provide regulatory certainty, proponents of the law are confident that it will boost the fintech industry and even create more competition to traditional banks at the same time [37].

\section{The Fintech Industry in Mexico}

Table 1 provides an overview of the fintech Industry in Mexico [41].

It could be stated that Mexico has a booming fintech industry, which experienced a $40 \%$ growth of startups within the last year. The 334 domestic fintech companies make Mexico a main driver to financial innovation in the region, second only to Brazil with 377 firms [41]. A study from 2017 revealed that new companies could take over 30\% of traditional banking market in Mexico within the next 10 years. Other experts even expect that Mexican fintech ecosystem will continue to grow at a fast pace and its market will eventually reach 70 billion USD by the end of 2018 [42].

Table 1. Fintech Industry in Mexico

\begin{tabular}{|l|c|}
\hline $\begin{array}{l}\text { Number of Fintech } \\
\text { Startups (August } \\
2018 \text { ) }\end{array}$ & 334 \\
\hline $\begin{array}{l}\text { Industry Growth in } \\
2017\end{array}$ & \multicolumn{1}{|c|}{$40 \%$} \\
\hline $\begin{array}{l}\text { Focus on Financial } \\
\text { Inclusion }\end{array}$ & $54 \%$ \\
\hline Main segments & $\begin{array}{l}\text { Payments \& Remittances Consumer } \\
\text { lending Enterprise Financial Management } \\
\text { Crowdfunding }\end{array}$ \\
\hline
\end{tabular}

\section{The Cryptocurrency Market}

Despite all the euphoric hype around cryptocurrencies and the promising future of blockchain companies, the cryptocurrency market is still in its infancy. Although the two Mexican exchange platforms Bitso and Volabit are leading the crypto currency exchange in Latin America, the Mexican market is less developed compared to the leading markets. Still, representatives of Bitso see a great growth potential in cryptocurrency use and trading [43].

With the new Fintech Law in force, Bitso strives for a closer collaboration with authorities (i.e. the central bank) and expects higher financial inclusion, reduced costs for remittance transactions and the "digitalization of money" [44].

Apart from the new law, the Mexican authorities have remained very quiet about their stance on blockchain and cryptocurrencies. Furthermore, there have been no ICOs
(Initial Coin Offerings) in Mexico yet. This is most probably due to the existing legal uncertainty, driven by the finance ministry's warning that ICOs could violate Mexican law [37]. Since many Mexicans do not use credit cards or bank accounts, digital payments for retailers in e-commerce get processed through convenience stores with systems like OXXO Pay [45]. Making use of this huge infrastructure, Bitso today offers cash deposits for bitcoin wallets as well as cash withdrawals at over 140'000 conveniences stores throughout the country [46]. This means that 140 '000 convenience stores were turned into cryptocurrency ATMs. In comparison, in the United States there are only 2'191 machines allowing direct cash transactions with cryptocurrency wallets [47]. Therefore, one can assume that Mexico has by far the largest density of access points to fund digital cryptocurrency wallets with cash worldwide.

As a result, Mexicans started to discover the benefits of this technology, primarily by using it for their everyday life. Since then, the awareness and adoption of Blockchain and Bitcoin have grown rapidly while the ecosystem has started growing. The customers segments at Bitso range from investors over people without credit cards using e-commerce for remittances [48].

Moreover, this existing infrastructure became even more important with the adoption of the new Fintech Law in March 2018, which legally recognized digital assets as a mean of payment and exchange. The combination of both technical and legal frameworks facilitates the use of crypto-currencies as remittance transactions. Hence, it creates robust opportunities for the blockchain industry, especially in a country where remittance transactions contribute more to its total GDP than oil revenue [49].

In 2017, payments floating into Mexico from abroad accounted for 28 billion USD, which represented $10 \%$ of Mexicans total GDP growth rate [50]. Moreover, remittances soared during the second quarter of this year and reached a new all-time high. As illustrated in Figure 1, only in the first quarter of 2018 the total amount of remittances exceeded 9 billion USD.

According to the World Bank [51], average global remittance costs amount to almost $7 \%$ today. Since the nature of blockchain allows for quasi instant transactions that can get executed at a small fraction of these costs, cross-border transactions with cryptocurrency could become an attractive alternative to traditional institutions like Western Union. The two popular Mexican exchanges Bitso and Volabit are already offering simple but useful applications, which can be used for this purpose. 


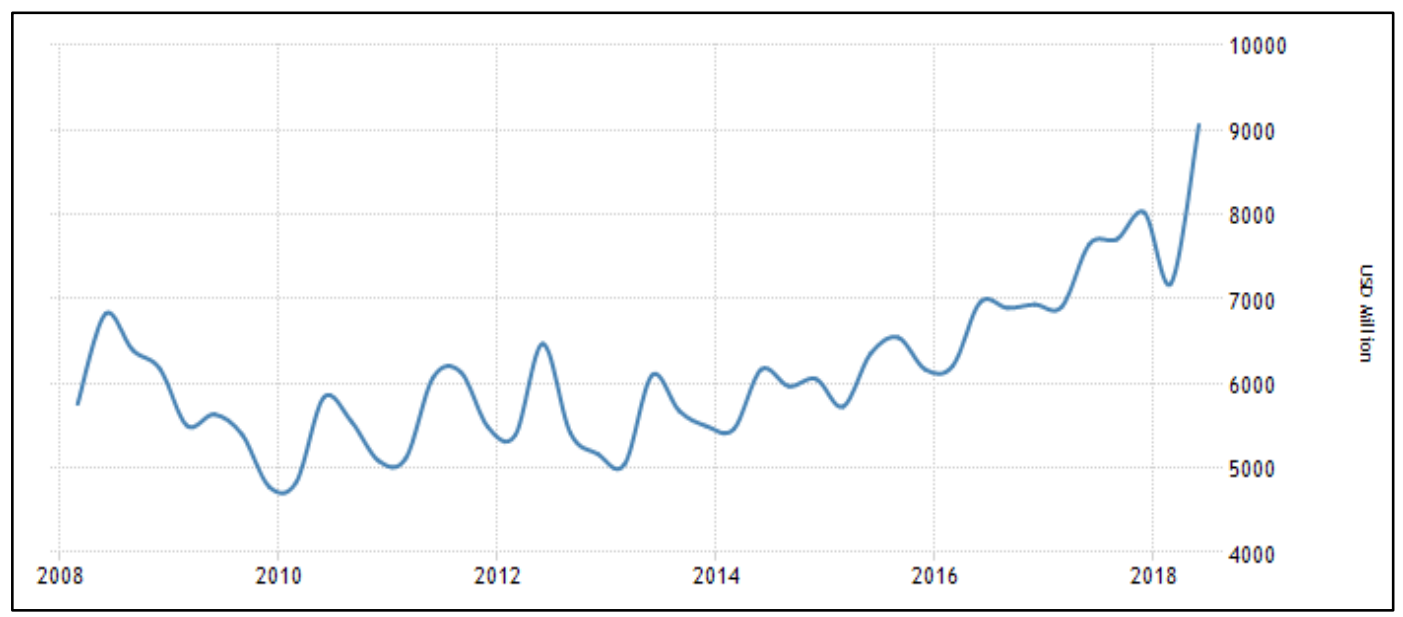

Figure 1. Mexico Remittances (Quarterly) [58]

\section{Talent and Education}

Compared to the United States where the blockchain development is flourishing, Mexico's blockchain development has started late and now struggles to retain talent since many Mexicans are tempted to work for tech-companies in the United States [52]. In response to the lack of local skill and to attract more talent, organizations like IBM, Tata Consultancy Services (TCS) Latin America and Telefónica Mexico aim to train the "technologically versatile" people in order that they can enhance their knowledge [52].

In addition, the recently launched Blockchain Academy Mexico offers blockchain courses for beginners, software engineers as well as business people. According to their website, the Blockchain Academy Mexico aims to contribute to the country's blockchain ecosystem by organizing courses as well as events [53].

\section{The Public Project "Blockchain HACKMX"}

Since September 2017, the Mexican government has been working on a blockchain project called "Blockchain HACKMX" [1]. The project initially arose from the recommendations of the World Economic Forum, which suggested that the use of blockchain technology might be beneficial in combating corruption. Blockchain is considered by the Mexican Government as a technical solution to enhance transparency and trust to eventually improve the processes of public procurement [55]. Blockchain HACKMX aims to "promote government digital innovation" and to address the issue of public contract corruption [1]. It involves five Government institutions and the state government of Jalisco. In collaboration with Campus Talent Mexico, they were creating a Talent Hackathon (i.e. contest) which encourages group of IT specialists to build blockchain solutions for public services such as tender process, identity management, certificates of deposits and public property register [1]. In total, 40 teams participated in the first hackathon whereby the majority of the projects of the ten finalists are in the area of identity management and public registers. The winning team created a blockchain-based solution for public tenders, which will allow for citizen participation.

Consequently, the Mexican government plans to test such a "smart" public tender on a blockchain [56]. According to reports, this pilot project will be based on the Ethereum blockchain, will use smart contracts and will operate without a cryptocurrency [56]. Residents will get the opportunity to take part in important decisions on a local, state and federal level. The voting process will happen in an anonymous way, preventing interest groups such as involved organizations, politicians and lobbyists from taking influence on voters - combating corruption at its roots. Another major benefit of this smart tender lies in the fact that citizens will always pursue the best option since they are the final beneficiaries [1].

Moreover, the long-term objective of the "Mexican Blockchain Initiative" is not only to develop separate projects such as this smart tender, but also to develop a national Mexican Blockchain for the public sector. This blockchain should be used as a foundation for further developments in the area of public property registers, identity management and certificates of deposits. Notably, the blockchain will not be fully public but rather a hybrid one, i.e. including public as well as private components. Since most public blockchains are not technically advanced yet to ensure a frictionless functionality, it becomes obvious that a public - private approach might be more appropriate at this stage [56]. Technically, this could mean that the block validation process will be conducted by private nodes, which are fully controlled by the government, universities or preselected companies.

\section{Discussion and Policy Implications}

As highlighted in the OECD survey presented in the case, the problems faced by Mexico are complex and often 
closely entangled with one another. One can argue that many issues such as corruption, weak rule of law or financial exclusion stem from the existing highly centralized system where power is concentrated in the hand of a few.

Blockchain technology as a decentralized system could act disruptively to the current state of power concentration. Splitting up the power of control among thousands of participants requires them to continuously agree on the data. This implies that every node stores the same copy of the data, which results in full transparency. Therefore, it becomes very difficult for a malicious actor to attack the system, i.e. trying to counterfeit or corrupt the data, since the other nodes normally ignore such attempts and the malicious actor is automatically excluded from the network In addition, the incentives used for reaching that consensus ensure that all nodes act in favor of the system, which makes it highly resistant to being corrupted in the first place.

However, Blockchain itself is by far no magic bullet for solving complex issues like corruption. Blockchain itself does not imply by default increasing efficiency or replacing trust. In terms of speed, costs, maintenance and upgrade simplicity, centralized databases still outperform blockchain. The one comparative advantage of blockchain though is decentralization. At the same time, decentralized systems, in particular public blockchains, make sense at most if they are correctly applied to environments where removing the single point of control in a system eliminates malicious practices like corruption and intransparency.

In conclusion, it could be argued that the main advantages associated with Blockchain are mainly driven by its decentralized nature of ensuring trust. Thus, the implementation of blockchain in areas like public procurement or public registers such as property and company registries could help in the fight against corruption $[2,57]$.

The Mexican Blockchain HACKMX" Project, for example, provides a technical solution to enhance transparency and trust to eventually improve the processes of public procurement by organizing public tender on a blockchain. Blockchain could thus help people in Mexico regain trust in the government and public institutions.

Nevertheless, it is important to note that using a private i.e. permissioned blockchain always reduces the factors of trust, such as integrity, transparency and immutability, and the degree of decentralization, such as privacy and reliability, within the system. Therefore, one can question the purpose of a permissioned blockchain since it is under the control of one certain authority or a certain, chosen consortium. Because all partners in the consortium are known and they might even share common interests, there would be no prevention of corruption possible. As a result, the use of blockchain technology would only complicate rather than help. One could argue that a serious approach towards combating corruption with blockchain would be to consider hybrid solutions, which can be incorporated into public blockchains.

It could be concluded that developing countries like Mexico could benefit if they modernize and digitalize their public services. The blockchain technology could facilitate this process thus fostering the transparency of entire government systems. However, the success of blockchain implementations is conditional on important decisions related to the type of blockchains (public, private or hybrid blockchains) and the public areas most appropriate for the introduction of blockchain. Moreover, a prerequisite for the realization of any potential benefits of blockchain is the existence of public awareness and education on blockchain.

\section{REFERENCES}

[1] Martínez Mancilla, Y. (2018). Blockchain HackMX. https://www.unece.org/fileadmin/DAM/cefact/cf_forums/2 017_Rome/PPTs/BlockChain/PM_05_Yolanda_Martinez_ Mancilla_Mexico_Blockchain_HĀCK̄MX.pdf

[2] Santiso, C. (2018). Will blockchain curb corruption? World Economic Forum.

[3] Vázquez Alanis, G. (2018). Blockchain and Bitcoin: The Situation in Mexico. Global Network Perspectives.

[4] He, D., Habermeier, K. Leckow, R., Haksar, V., Kashima, M., Kyriakos-Saad, N., Verdugo Yepes, C. (2016). Virtual Currencies and Beyond: Initial Considerations. IMF Staff Discussion Notes, 16(03), 1.

[5] Kostarev, G. (2017). Review of blockchain consensus mechanisms [Medium Blog Post]. Waves Platform.

[6] Bradley, R. (2017). Blockchain explained in under 100 words. Deloitte.

[7] Tapscott, D., \& Tapscott, A. (2016). Blockchain revolution: how the technology behind bitcoin is changing money, business, and the world. New York: Penguin.

[8] Nakamoto, S. (2008). Bitcoin: A Peer-to-Peer Electronic Cash System. https://bitcoin.org/bitcoin.pdf

[9] Chohan, U. W. (2017). The Double Spending Problem and Cryptocurrencies. SSRN Scholarly Paper No. ID 3090174. Rochester, NY: Social Science Research Network.

[10] Christidis, K., \& Devetsikiotis, M. (2016). Blockchains and Smart Contracts for the Internet of Things. IEEE Access, 4 , 2292-2303.

[11] Gupta, V. (2017). The Promise of Blockchain Is a World Without Middlemen. Harvard Business Review.

[12] Waves platform. (2018). The Future of DApps. Steemit Blog Post.

[13] Blockchain Taskforce Switzerland. (2018). Strengthening the blockchain in Switzerland. White paper.

[14] Bettinger M. (2018, May 5). Personal communication. 
[15] Song, J. (2018). Why blockchain is hard. Medium.com.

[16] Jia, Ch. (2016). What Is the True Meaning of "Decentralization" in Blockchain Technology? Bitcoinmagazine.com.

[17] The Economist. (2018). Why bitcoin uses so much energy. The Economist.

[18] Digiconomist. (2018). Bitcoin Energy Consumption Index.

[19] CIA. (2016). The World Factbook.

[20] Antonopoulos, A. M. (2017). Mastering Bitcoin: Programming the Open Blockchain. Kindle Edition.

[21] Domingo, C. (2017). The Bitcoin vs Visa Electricity Consumption Fallacy. Hackernoon.

[22] Chavez-Dreyfuss, G. (2018). About $\$ 1.2$ billion in cryptocurrency stolen since 2017. Reuters.

[23] OECD. (2017). OECD Economic Survey of Mexico 2017.

[24] Estevez, D. (2017). Better Year for Mexico's Billionaires. Forbes.

[25] World Bank. (2016). Poverty and Equity Database.

[26] Keeley, B. (2015). Income Inequality: The Gap between Rich and Poor. OECD Survey.

[27] OECD. (2016). Earnings and wages - Average wages. OECD Data.

[28] World Bank. (2016). Poverty and Equity Database

[29] Comisión Nacional Bancaria Y De Valores. (2016). ENIF 2015: Encuesta nacional de inclusión financiera [National survey about financial inclusion]. Online available from https://www.cnbv.gob.mx/Inclusi\%C3\%B3n/Documents/E ncuesta $\% 20$ Nacional $\% 20 \mathrm{de} \% 20 \mathrm{IF} / \mathrm{Cuadr} \% \mathrm{C} 3 \% \mathrm{ADptico} \%$ 202016\%20(impresi \%C3\%B3n\%20carta).pdf

[30] Center for International Private Enterprise \& International Real Property Foundation. (2014). Access to Credit Survey.

[31] Solo, T. M. (2008). Financial exclusion in Latin America or the social costs of not banking the urban poor. Environment and Urbanization, 20(1), 47-66.

[32] De Soto, H. (2000). The mystery of capital: why capitalism triumphs in the West and fails everywhere else. New York, NY: Basic Books.

[33] Transparency International. (2018). Mexico Report.

[34] Vice, M., \& Chwe, H. (2017). Mexicans are downbeat about their country's direction. Pew Research Center.

[35] Bjørnskov, C. (2006). How does social trust affect economic growth? Aarhus: Aarhus School of Business, Department of Economics Working Paper.

[36] Guiso, L., Sapienza, P., \& Zingales, L. (2006). Does Culture Affect Economic Outcomes? American Economic Association, 20(2), 23-48.

[37] Reuters. (2018, March 1). Mexico financial technology law passes final hurdle in Congress.

[38] Mexican Fintech Law. (2018). Law to Regulate Financial Technology Institutions.
[39] García, C. (2018, July 31). Entran en vigencia nuevas regulaciones del Banco de Mexico sobre depósitos en casas de cambio de criptoactivos. Cripto Noticias. https://www.criptonoticias.com/regulacion/entran-vigencianuevas-regulaciones-banco-mexico-depositos-casas-cambio -criptoactivos/

[40] Butty, J. (2018). Fintech Regulations - The Mexican Case. Seedstars.

[41] Finnovista. (2018). Mexico exceeds the barrier of 300 Fintech startups and reinforces its position as the second most important Fintech ecosystem in Latin America. Finnovista.

[42] Kern, M. (2018). Mexico's Fintech Industry Is Booming. Safehaven.

[43] Quintero, L. (2018, February 20). Mexico, cryptocurrency leader in Latin America. El Universal.

[44] Dabdoub, F. V. (2018, March 12). Ley Fintech: Implicaciones para Bitso y la relación con sus usuarios. https://blog.bitso.com/ley-fintech-implicaciones-para-bitsoy-la-relaci $\% \mathrm{C} 3 \% \mathrm{~B} 3 \mathrm{n}$-con-sus-usuarios-57f5acfabc 40

[45] OXXO, Conekta, \& Femsa. (2017). OXXO PAY: The New Digital Payment Solution for Retailers. FEMSA.

[46] Bitso. (2018, August 21). How to fund in cash via Oxxo? https://help.bitso.com/en/support/solutions/articles/1000166 720-tutorial-how-to-fund-in-cash-via-oxxo-

[47] Coin ATM Radar. (2018). Bitcoin ATM United States. https://coinatmradar.com/country/226/bitcoin-atm-united-st ates/

[48] Connell, J. (2017, January 2). Why Volume Is Exploding at Mexican Bitcoin Exchange Bitso. Bitcoin.com

[49] NBC News. (2016, February 3). Mexico Got More Money from Remittances Than from Oil Revenues in 2015. NBC News.

[50] Hernández, L. (2018, January 30). Remesas aportan décima parte del crecimiento del PIB del país. El Financiero. http://www.elfinanciero.com.mx/economia/remesas-aporta n-decima-parte-del-crecimiento-del-pib-del-pais.html

[51] World Bank. (2018). Remittance Prices Worldwide. World Bank Working Paper (No. 26).

[52] Kendall, M. (2018, April 25). Mexico "Three-Out-Of-Ten" on the Global Blockchain Scale, Despite Robust Opportunities. Nearshoreamericas.com.

[53] Blockchain Academy México. (2018). Cursos teóricos y de programación. https://blockchainacademy.mx/cursos

[54] Telefónica. (2018). Mexico City Blockchain Bootcamp. http://www.openfuture.org/es/event/mexico-city-blockchai n-bootcamp_1

[55] Malwa, S. (2018, April 9). The Mexican Government To Pilot Run A Blockchain System. BTCManager.

[56] Riquelme, R. (2018, July 27). Gobierno federal realizará el primer caso real de licitación con blockchain en agosto. El economista.

https://www.eleconomista.com.mx/tecnologia/Gobierno-fe deral-realizara-el-primer-caso-real-de-licitacion-con-blockc 
hain-en-agosto-20180727-0035.html

[57] Nelson, A. (2018). De Soto Inc.: Where Eminent Domain Meets the Blockchain. Bitcoinmagazine.com.
[58] Trading Economics \& Transparency International. (2018). Mexico Corruption Rank 1995-2018. https://tradingeconom ics.com/mexico/corruption-rank. 